\title{
$\Phi$-Admissible singular operators and their commutators on vanishing generalized Orlicz-Morrey spaces
}

\author{
Vagif S Guliyev ${ }^{1,2^{*}}$, Fatih Deringoz ${ }^{1}$ and Javanshir J Hasanov²
}

\section{"Correspondence:}

vagif@guliyev.com

'Department of Mathematics, Ahi

Evran University, Kirsehir, Turkey

${ }^{2}$ Institute of Mathematics and

Mechanics, Baku, Azerbaijan

\begin{abstract}
We study the boundedness of $\Phi$-admissible singular operators and their commutators on vanishing generalized Orlicz-Morrey spaces $V M_{\Phi, \varphi}\left(\mathbb{R}^{n}\right)$ including their weak versions. These conditions are satisfied by most of the operators in harmonic analysis, such as the Hardy-Littlewood maximal operator, the Calderón-Zygmund singular integral operator and so on. In all the cases the conditions for the boundedness are given in terms of Zygmund-type integral inequalities on weights $\varphi(x, r)$ without assuming any monotonicity property of $\varphi(x, r)$ on $r$.

MSC: 42B20; 42B25; 42B35; 46E30

Keywords: vanishing generalized Orlicz-Morrey space; $\Phi$-admissible singular operators; Hardy-Littlewood maximal operator; Calderón-Zygmund singular integral operator; commutator; $\mathrm{BMO}$
\end{abstract}

\section{Introduction}

As is well known Morrey [1] introduced the classical Morrey spaces to investigate the local behavior of solutions to second order elliptic partial differential equations (PDE). We recall its definition as

$$
M_{p, \lambda}\left(\mathbb{R}^{n}\right)=\left\{f \in L_{p}^{\text {loc }}\left(\mathbb{R}^{n}\right):\|f\|_{M_{p, \lambda}}:=\sup _{x \in \mathbb{R}^{n}, r>0} r^{-\frac{\lambda}{p}}\|f\|_{L_{p}(B(x, r))}<\infty\right\},
$$

where $0 \leq \lambda \leq n, 1 \leq p<\infty$. Here and everywhere in the sequel $B(x, r)$ stands for the ball in $\mathbb{R}^{n}$ of radius $r$ centered at $x$. Let $|B(x, r)|$ be the Lebesgue measure of the ball $B(x, r)$ and $|B(x, r)|=v_{n} r^{n}$, where $v_{n}=|B(0,1)| \cdot M_{p, \lambda}\left(\mathbb{R}^{n}\right)$ was an expansion of $L_{p}\left(\mathbb{R}^{n}\right)$ in the sense that $M_{p, 0}\left(\mathbb{R}^{n}\right)=L_{p}\left(\mathbb{R}^{n}\right)$. We also denote by $W M_{p, \lambda} \equiv W M_{p, \lambda}\left(\mathbb{R}^{n}\right)$ the weak Morrey space of all functions $f \in W L_{p}^{\text {loc }}\left(\mathbb{R}^{n}\right)$ for which

$$
\|f\|_{W M_{p, \lambda}}=\sup _{x \in \mathbb{R}^{n}, r>0} r^{-\frac{\lambda}{p}}\|f\|_{W L_{p}(B(x, r))}<\infty,
$$

where $W L_{p}(B(x, r))$ denotes the weak $L_{p}$-space.

Morrey found that many properties of solutions to PDE can be attributed to the boundedness of some operators on Morrey spaces. Maximal functions and singular integrals play a key role in harmonic analysis, since maximal functions could control crucial quantitative

○2014 Guliyev et al.; licensee Springer. This is an Open Access article distributed under the terms of the Creative Commons Attribution License (http://creativecommons.org/licenses/by/2.0), which permits unrestricted use, distribution, and reproduction in any medium, provided the original work is properly cited. 
information concerning the given functions, despite their larger size, while singular integrals, with the Hilbert transform as their prototype, nowadays are intimately connected with PDE, operator theory, and other fields.

Orlicz spaces, introduced in [2,3], are generalizations of Lebesgue spaces $L_{p}$. They are useful tools in harmonic analysis and its applications. For example, the Hardy-Littlewood maximal operator is bounded on $L_{p}$ for $1<p<\infty$, but not on $L_{1}$. Using Orlicz spaces, we can investigate the boundedness of the maximal operator near $p=1$ more precisely (see $[4,5]$ and $[6])$.

Let $f \in L_{1}^{\text {loc }}\left(\mathbb{R}^{n}\right)$. The Hardy-Littlewood maximal function of $f$ is defined by

$$
M f(x)=\sup _{r>0} \frac{1}{|B(x, r)|} \int_{B(x, r)}|f(y)| d y .
$$

The Calderón-Zygmund singular integral operator is defined by

$$
K f(x)=\int_{\mathbb{R}^{n}} k(x, y) f(y) d y
$$

and bounded on $L_{2}\left(\mathbb{R}^{n}\right)$, where $K(x, y)$ is a 'standard singular kernel', that is, a continuous function defined on $\left\{(x, y) \in \mathbb{R}^{n} \times \mathbb{R}^{n}: x \neq y\right\}$ and satisfying the estimates

$$
\begin{aligned}
& |k(x, y)| \leq C|x-y|^{-n} \quad \text { for all } x \neq y, \\
& |k(x, y)-k(x, z)| \leq C \frac{|y-z|^{\sigma}}{|x-y|^{n+\sigma}}, \quad \sigma>0, \text { if }|x-y|>2|y-z|, \\
& |k(x, y)-k(\xi, y)| \leq C \frac{|x-\xi|^{\sigma}}{|x-y|^{n+\sigma}}, \quad \sigma>0, \text { if }|x-y|>2|x-\xi| .
\end{aligned}
$$

It is well known that the maximal and singular integral operators play an important role in harmonic analysis (see $[7,8])$.

Remark 1.1 The Calderón-Zygmund singular integral operators $K$ are $L_{2}$ bounded and expressed as (1.1) for all $f \in C_{\text {comp }}^{\infty}\left(\mathbb{R}^{n}\right)$, with standard kernel $k$. Then one can prove that $K$ is of weak type $(1,1)$ and type $(p, p), 1<p<\infty$, for $f \in C_{\text {comp }}^{\infty}\left(\mathbb{R}^{n}\right)$, and then $K$ is uniquely extended to an $L_{p}$-bounded operator by the density of $C_{\text {comp }}^{\infty}\left(\mathbb{R}^{n}\right)$ in $L_{p}\left(\mathbb{R}^{n}\right)$. On the other hand, $C_{\text {comp }}^{\infty}\left(\mathbb{R}^{n}\right)$ is not dense in Morrey spaces in general. Therefore, we need to give a precise definition of $K f$ for the function $f$ in Morrey spaces, for example,

$$
K f(x)=K\left(f \chi_{2 B}\right)+\int_{\mathbb{R}^{n} \backslash(2 B)} k(x, y) f(y) d y,
$$

for some ball $B$ which contains $x$, proving the absolute convergence of the integral in the second term and the independence of the choice of the ball $B$ (see $[9,10]$ for example). Also, $C_{\text {comp }}^{\infty}\left(\mathbb{R}^{n}\right)$ is dense in the Orlicz spaces $L_{\Phi}\left(\mathbb{R}^{n}\right)$ if and only if $\Phi$ satisfies the $\Delta_{2}$ condition.

The main purpose of this paper is to find sufficient conditions on general Young function $\Phi$ and functions $\varphi_{1}, \varphi_{2}$ which ensure the boundedness of the sublinear operators generated by singular integral operators from vanishing generalized Orlicz-Morrey spaces $V M_{\Phi, \varphi_{1}}\left(\mathbb{R}^{n}\right)$ to another $V M_{\Phi, \varphi_{2}}\left(\mathbb{R}^{n}\right)$, from $V M_{\Phi, \varphi_{1}}\left(\mathbb{R}^{n}\right)$ to vanishing weak generalized 
Orlicz-Morrey spaces $V W M_{\Phi, \varphi_{2}}\left(\mathbb{R}^{n}\right)$ and the boundedness of the commutator of the sublinear operators from $V M_{\Phi, \varphi_{1}}\left(\mathbb{R}^{n}\right)$ to $V M_{\Phi, \varphi_{2}}\left(\mathbb{R}^{n}\right)$.

There are several kinds of Orlicz-Morrey spaces in the literature. The first kind is due to Nakai [9] and the second kind is due to Sawano et al. [11]. Our definition (see [12]) should be called 'generalized Orlicz-Morrey space of the third kind'. For the boundedness of the operators of harmonic analysis on Orlicz-Morrey spaces, see also [10-19]. For details see Remark 9 in [13] and references therein.

By $A \lesssim B$ we mean that $A \leq C B$ with some positive constant $C$ independent of appropriate quantities. If $A \lesssim B$ and $B \lesssim A$, we write $A \approx B$ and say that $A$ and $B$ are equivalent.

\section{Preliminaries}

We recall the definition of Young functions.

Definition 2.1 A function $\Phi:[0,+\infty) \rightarrow[0, \infty]$ is called a Young function if $\Phi$ is convex, left-continuous, $\lim _{r \rightarrow+0} \Phi(r)=\Phi(0)=0$, and $\lim _{r \rightarrow+\infty} \Phi(r)=\infty$.

From the convexity and $\Phi(0)=0$ it follows that any Young function is increasing. If there exists $s \in(0,+\infty)$ such that $\Phi(s)=+\infty$, then $\Phi(r)=+\infty$ for $r \geq s$.

Let $\mathcal{Y}$ be the set of all Young functions $\Phi$ such that

$$
0<\Phi(r)<+\infty \text { for } 0<r<+\infty
$$

If $\Phi \in \mathcal{Y}$, then $\Phi$ is absolutely continuous on every closed interval in $[0,+\infty)$ and bijective from $[0,+\infty)$ to itself.

Definition 2.2 (Orlicz space) For a Young function $\Phi$, the set

$$
L_{\Phi}\left(\mathbb{R}^{n}\right)=\left\{f \in L_{1}^{\text {loc }}\left(\mathbb{R}^{n}\right): \int_{\mathbb{R}^{n}} \Phi(k|f(x)|) d x<+\infty \text { for some } k>0\right\}
$$

is called Orlicz space. If $\Phi(r)=r^{p}, 1 \leq p<\infty$, then $L_{\Phi}\left(\mathbb{R}^{n}\right)=L_{p}\left(\mathbb{R}^{n}\right)$. If $\Phi(r)=0(0 \leq r \leq 1)$ and $\Phi(r)=\infty(r>1)$, then $L_{\Phi}\left(\mathbb{R}^{n}\right)=L_{\infty}\left(\mathbb{R}^{n}\right)$. The space $L_{\Phi}^{\text {loc }}\left(\mathbb{R}^{n}\right)$ endowed with the natural topology is defined as the set of all functions $f$ such that $f \chi_{B} \in L_{\Phi}\left(\mathbb{R}^{n}\right)$ for all balls $B \subset \mathbb{R}^{n}$. We refer to the books [20-22] for the theory of Orlicz spaces.

$L_{\Phi}\left(\mathbb{R}^{n}\right)$ is a Banach space with respect to the norm

$$
\|f\|_{L_{\Phi}}=\inf \left\{\lambda>0: \int_{\mathbb{R}^{n}} \Phi\left(\frac{|f(x)|}{\lambda}\right) d x \leq 1\right\} .
$$

We note that, from the Fatou lemma,

$$
\int_{\mathbb{R}^{n}} \Phi\left(\frac{|f(x)|}{\|f\|_{L_{\Phi}}}\right) d x \leq 1 .
$$

For a measurable set $\Omega \subset \mathbb{R}^{n}$, a measurable function $f$, and $t>0$, let

$$
m(\Omega, f, t)=|\{x \in \Omega:|f(x)|>t\}| .
$$

In the case $\Omega=\mathbb{R}^{n}$, we shortly denote it by $m(f, t)$. 
Definition 2.3 The weak Orlicz space

$$
W L_{\Phi}\left(\mathbb{R}^{n}\right):=\left\{f \in L_{1}^{\operatorname{loc}}\left(\mathbb{R}^{n}\right):\|f\|_{W L_{\Phi}}<+\infty\right\}
$$

is defined by the norm

$$
\|f\|_{W L_{\Phi}}=\inf \left\{\lambda>0: \sup _{t>0} \Phi(t) m\left(\frac{f}{\lambda}, t\right) \leq 1\right\} .
$$

For a Young function $\Phi$ and $0 \leq s \leq+\infty$, let

$$
\Phi^{-1}(s)=\inf \{r \geq 0: \Phi(r)>s\} \quad(\inf \emptyset=+\infty) .
$$

If $\Phi \in \mathcal{Y}$, then $\Phi^{-1}$ is the usual inverse function of $\Phi$. We note that

$$
\Phi\left(\Phi^{-1}(r)\right) \leq r \leq \Phi^{-1}(\Phi(r)) \quad \text { for } 0 \leq r<+\infty .
$$

A Young function $\Phi$ is said to satisfy the $\Delta_{2}$-condition, denoted by $\Phi \in \Delta_{2}$, if

$$
\Phi(2 r) \leq k \Phi(r) \quad \text { for } r>0
$$

for some $k>1$. If $\Phi \in \Delta_{2}$, then $\Phi \in \mathcal{Y}$. A Young function $\Phi$ is said to satisfy the $\nabla_{2}$-condition, denoted also by $\Phi \in \nabla_{2}$, if

$$
\Phi(r) \leq \frac{1}{2 k} \Phi(k r), \quad r \geq 0
$$

for some $k>1$. The function $\Phi(r)=r$ satisfies the $\Delta_{2}$-condition but does not satisfy the $\nabla_{2}$-condition. If $1<p<\infty$, then $\Phi(r)=r^{p}$ satisfies both conditions. The function $\Phi(r)=$ $e^{r}-r-1$ satisfies the $\nabla_{2}$-condition but does not satisfy the $\Delta_{2}$-condition.

For a Young function $\Phi$, the complementary function $\widetilde{\Phi}(r)$ is defined by

$$
\widetilde{\Phi}(r)= \begin{cases}\sup \{r s-\Phi(s): s \in[0, \infty)\}, & r \in[0, \infty) \\ +\infty, & r=+\infty\end{cases}
$$

The complementary function $\widetilde{\Phi}$ is also a Young function and $\widetilde{\Phi}=\Phi$. If $\Phi(r)=r$, then $\widetilde{\Phi}(r)=0$ for $0 \leq r \leq 1$ and $\widetilde{\Phi}(r)=+\infty$ for $r>1$. If $1<p<\infty, 1 / p+1 / p^{\prime}=1$ and $\Phi(r)=r^{p} / p$, then $\widetilde{\Phi}(r)=r^{p^{\prime}} / p^{\prime}$. If $\Phi(r)=e^{r}-r-1$, then $\widetilde{\Phi}(r)=(1+r) \log (1+r)-r$. Note that $\Phi \in \nabla_{2}$ if and only if $\widetilde{\Phi} \in \Delta_{2}$. It is well known that

$$
r \leq \Phi^{-1}(r) \widetilde{\Phi}^{-1}(r) \leq 2 r \quad \text { for } r \geq 0 .
$$

Note that Young functions satisfy the properties

$$
\left\{\begin{array} { l } 
{ \Phi ( \alpha t ) \leq \alpha \Phi ( t ) , \quad \text { if } 0 \leq \alpha \leq 1 , } \\
{ \Phi ( \alpha t ) \geq \alpha \Phi ( t ) , \quad \text { if } \alpha > 1 }
\end{array} \text { and } \left\{\begin{array}{ll}
\Phi^{-1}(\alpha t) \geq \alpha \Phi^{-1}(t), & \text { if } 0 \leq \alpha \leq 1 \\
\Phi^{-1}(\alpha t) \leq \alpha \Phi^{-1}(t), & \text { if } \alpha>1
\end{array}\right.\right.
$$

The following analog of the Hölder inequality is well known; see [23]. 
Theorem 2.4 [23] For a Young function $\Phi$ and its complementary function $\widetilde{\Phi}$, the following inequality is valid:

$$
\|f g\|_{L_{1}\left(\mathbb{R}^{n}\right)} \leq 2\|f\|_{L_{\Phi}}\|g\|_{L_{\widetilde{\Phi}}}
$$

The following lemma is valid. See, for example [19, 24, 25].

Lemma 2.5 Let $\Phi$ be a Young function and B a set in $\mathbb{R}^{n}$ with finite Lebesgue measure. Then

$$
\left\|\chi_{B}\right\|_{W L_{\Phi}\left(\mathbb{R}^{n}\right)}=\left\|\chi_{B}\right\|_{L_{\Phi}\left(\mathbb{R}^{n}\right)}=\frac{1}{\Phi^{-1}\left(|B|^{-1}\right)} .
$$

In the next sections where we prove our main estimates, we use the following lemma, which follows from Theorem 2.4, Lemma 2.5, and (2.2).

Lemma 2.6 For a Young function $\Phi$ and $B=B(x, r)$, the following inequality is valid:

$$
\|f\|_{L_{1}(B)} \leq 2|B| \Phi^{-1}\left(|B|^{-1}\right)\|f\|_{L_{\Phi}(B)} .
$$

Let $T$ be a sublinear operator, that is, $|T(f+g)| \leq|T f|+|T g|$.

Definition 2.7 ( $\Phi$-admissible singular operator) Let $\Phi$ any Young function. A sublinear operator $T$ will be called $\Phi$-admissible singular operator, if:

(1) $T$ satisfies the size condition of the form

$$
\chi_{B(x, r)}(z)\left|T\left(f \chi_{\mathbb{R}^{n} \backslash B(x, 2 r)}\right)(z)\right| \leq C \chi_{B(x, r)}(z) \int_{\mathbb{R}^{n} \backslash B(x, 2 r)} \frac{|f(y)|}{|y-z|^{n}} d y
$$

for $x \in \mathbb{R}^{n}$ and $r>0$;

(2) $T$ is bounded in $L_{\Phi}\left(\mathbb{R}^{n}\right)$.

In the case $\Phi(r)=r^{p}, 1<p<\infty$, the $\Phi$-admissible singular operator will be called the $p$-admissible singular operator.

Definition 2.8 (Weak $\Phi$-admissible singular operator) Let $\Phi$ any Young function. A sublinear operator $T$ will be called the weak $\Phi$-admissible singular operator, if:

(1) $T$ satisfies the size condition (2.3).

(2) $T$ is bounded from $L_{\Phi}\left(\mathbb{R}^{n}\right)$ to the weak $W L_{\Phi}\left(\mathbb{R}^{n}\right)$.

In the case $\Phi(r)=r^{p}, 1 \leq p<\infty$. the weak $\Phi$-admissible singular operator will be called weak $p$-admissible singular operator.

Remark 2.9 Note that in [14], $\Phi$-admissible singular operators and weak $\Phi$-admissible singular operators were introduced and their boundedness on generalized Orlicz-Morrey spaces was studied. Also in [26], $p$-admissible singular operators were introduced and their boundedness on vanishing generalized Morrey spaces was studied. 
Definition 2.10 (Generalized Orlicz-Morrey space) Let $\varphi(x, r)$ be a positive measurable function on $\mathbb{R}^{n} \times(0, \infty)$ and $\Phi$ any Young function. We denote by $M_{\Phi, \varphi}\left(\mathbb{R}^{n}\right)$ the generalized Orlicz-Morrey space, the space of all functions $f \in L_{\Phi}^{\text {loc }}\left(\mathbb{R}^{n}\right)$ with finite quasinorm

$$
\|f\|_{M_{\Phi, \varphi}}=\sup _{x \in \mathbb{R}^{n}, r>0} \varphi(x, r)^{-1}\|f\|_{L_{\Phi}(B(x, r))} .
$$

Also by $W M_{\Phi, \varphi}\left(\mathbb{R}^{n}\right)$ we denote the weak generalized Orlicz-Morrey space of all functions $f \in W L_{\Phi}^{\text {loc }}\left(\mathbb{R}^{n}\right)$ for which

$$
\|f\|_{W M_{\Phi, \varphi}}=\sup _{x \in \mathbb{R}^{n}, r>0} \varphi(x, r)^{-1}\|f\|_{W L_{\Phi}(B(x, r))}<\infty .
$$

According to this definition, we recover the generalized Morrey space $M_{p, \varphi}$ and weak generalized Morrey space $W M_{p, \varphi}$ under the choice $\Phi(r)=r^{p}, 1 \leq p<\infty$ :

$$
M_{p, \varphi}=\left.M_{\Phi, \varphi}\right|_{\Phi(r)=r^{p}}, \quad W M_{p, \varphi}=\left.W M_{\Phi, \varphi}\right|_{\Phi(r)=r^{p}} .
$$

The vanishing generalized Morrey space $V M_{p, \varphi}\left(\mathbb{R}^{n}\right)$ which was introduced and studied by Samko [26] is defined as follows.

Definition 2.11 (Vanishing generalized Morrey space) Let $\varphi(x, r)$ be a positive measurable function on $\mathbb{R}^{n} \times(0, \infty)$ and $1 \leq p<\infty$. The vanishing generalized Morrey space $V M_{p, \varphi}\left(\mathbb{R}^{n}\right)$ is defined as the space of functions $f \in M_{p, \varphi}\left(\mathbb{R}^{n}\right)$ such that

$$
\lim _{r \rightarrow 0} \sup _{x \in \mathbb{R}^{n}} \varphi(x, r)^{-1} \int_{B(x, r)}|f(y)|^{p} d y=0 .
$$

Extending the definition of vanishing generalized Morrey spaces to the case of OrliczMorrey spaces, we introduce the following definitions.

Definition 2.12 (Vanishing generalized Orlicz-Morrey space) The vanishing generalized Orlicz-Morrey space $V M_{\Phi, \varphi}\left(\mathbb{R}^{n}\right)$ is defined as the space of functions $f \in M_{\Phi, \varphi}\left(\mathbb{R}^{n}\right)$ such that

$$
\lim _{r \rightarrow 0} \sup _{x \in \mathbb{R}^{n}} \varphi(x, r)^{-1}\|f\|_{L_{\Phi}(B(x, r))}=0 .
$$

Definition 2.13 (Vanishing weak generalized Orlicz-Morrey space) The vanishing weak generalized Orlicz-Morrey space $V W M_{\Phi, \varphi}\left(\mathbb{R}^{n}\right)$ is defined as the space of functions $f \in$ $W M_{\Phi, \varphi}\left(\mathbb{R}^{n}\right)$ such that

$$
\lim _{r \rightarrow 0} \sup _{x \in \mathbb{R}^{n}} \varphi(x, r)^{-1}\|f\|_{W L_{\Phi}(B(x, r))}=0 .
$$

If we choose $\Phi(r)=r^{p}, 1 \leq p<\infty$ at Definition 2.12, we get Definition 2.11. The vanishing Morrey space $V M_{p, \lambda}\left(\mathbb{R}^{n}\right)$ of the classical Morrey space $M_{p, \lambda}\left(\mathbb{R}^{n}\right)$ was introduced by Vitanza in [27] and applied there to obtain a regularity result for elliptic partial differential equations. Later in [28] Vitanza proved an existence theorem for a Dirichlet problem, 
under weaker assumptions than those introduced by Miranda in [29], and a $W^{3,2}$ regularity result assuming that the partial derivatives of the coefficients of the highest and lower order terms belong to a vanishing Morrey space depending on the dimension. Also Ragusa [30] proved a sufficient condition for commutators of fractional integral operators to belong to vanishing Morrey spaces $V M_{p, \lambda}\left(\mathbb{R}^{n}\right)$. About commutator operators in vanishing Morrey spaces see the papers [30, 31].

Everywhere in the sequel we assume that

$$
\lim _{r \rightarrow 0} \frac{1}{\Phi^{-1}\left(r^{-n}\right) \inf _{x \in \mathbb{R}^{n}} \varphi(x, r)}=0
$$

and

$$
\sup _{0<r<\infty} \frac{1}{\Phi^{-1}\left(r^{-n}\right) \inf _{x \in \mathbb{R}^{n}} \varphi(x, r)}<\infty
$$

which makes the spaces $V M_{\Phi, \varphi}\left(\mathbb{R}^{n}\right)$ and $V W M_{\Phi, \varphi}\left(\mathbb{R}^{n}\right)$ non-trivial, because bounded functions with compact support belong then to this space.

The spaces $V M_{\Phi, \varphi}\left(\mathbb{R}^{n}\right)$ and $W V M_{\Phi, \varphi}\left(\mathbb{R}^{n}\right)$ are Banach spaces with respect to the norm

$$
\begin{aligned}
& \|f\|_{V M_{\Phi, \varphi}} \equiv\|f\|_{M_{\Phi, \varphi}}=\sup _{x \in \mathbb{R}^{n}, r>0} \varphi(x, r)^{-1}\|f\|_{L_{\Phi}(B(x, r))}, \\
& \|f\|_{V W M_{\Phi, \varphi}} \equiv\|f\|_{W M_{\Phi, \varphi}}=\sup _{x \in \mathbb{R}^{n}, r>0} \varphi(x, r)^{-1}\|f\|_{W L_{\Phi}(B(x, r))},
\end{aligned}
$$

respectively. The spaces $V M_{p, \varphi}\left(\mathbb{R}^{n}\right)$ and $V W M_{p, \varphi}\left(\mathbb{R}^{n}\right)$ are closed subspaces of the Banach spaces $M_{p, \varphi}\left(\mathbb{R}^{n}\right)$ and $W M_{p, \varphi}\left(\mathbb{R}^{n}\right)$, respectively, which may be shown by standard means.

\section{$3 \Phi$-Admissible singular operators in the spaces $V M_{\Phi, \varphi}$}

In this section, sufficient conditions on $\varphi$ for the boundedness of the $\Phi$-admissible singular operator $T$ in vanishing generalized Orlicz-Morrey spaces $V M_{\Phi, \varphi}\left(\mathbb{R}^{n}\right)$ are obtained.

The known boundedness statement for the Hardy-Littlewood maximal operator $M$ and the Calderón-Zygmund singular integral operators $K$ in Orlicz spaces runs as follows. For details of these results see [12].

Theorem 3.1 [5,20] Let $\Phi$ any Young function. Then the maximal operator $M$ is bounded from $L_{\Phi}\left(\mathbb{R}^{n}\right)$ to $W L_{\Phi}\left(\mathbb{R}^{n}\right)$ and for $\Phi \in \nabla_{2}$ bounded in $L_{\Phi}\left(\mathbb{R}^{n}\right)$.

Theorem 3.2 [20,32] Let $\Phi$ be a Young function. If $\Phi \in \Delta_{2} \cap \nabla_{2}$, then the operator $K$ is bounded on $L_{\Phi}\left(\mathbb{R}^{n}\right)$ and if $\Phi \in \Delta_{2}$, then the operator $K$ is bounded from $L_{\Phi}\left(\mathbb{R}^{n}\right)$ to $W L_{\Phi}\left(\mathbb{R}^{n}\right)$.

The following lemma was a generalization of the Guliyev lemma for Orlicz spaces [33$35]$, and it was proved in [14].

Lemma 3.3 Let $\Phi$ any Young function and $f \in L_{\Phi}^{\text {loc }}\left(\mathbb{R}^{n}\right), B=B\left(x_{0}, r\right), x_{0} \in \mathbb{R}^{n}$, and $r>0$. Then for the $\Phi$-admissible singular operator $T$ the following inequality is valid:

$$
\|T f\|_{L_{\Phi}(B)} \lesssim \frac{1}{\Phi^{-1}\left(r^{-n}\right)} \int_{2 r}^{\infty}\|f\|_{L_{\Phi}\left(B\left(x_{0}, t\right)\right)} \Phi^{-1}\left(t^{-n}\right) \frac{d t}{t},
$$


and for the weak $\Phi$-admissible singular operator $T$ the following inequality is valid:

$$
\|T f\|_{W L_{\Phi}(B)} \lesssim \frac{1}{\Phi^{-1}\left(r^{-n}\right)} \int_{2 r}^{\infty}\|f\|_{L_{\Phi}\left(B\left(x_{0}, t\right)\right)} \Phi^{-1}\left(t^{-n}\right) \frac{d t}{t} .
$$

By using Lemma 3.3 the following statement was proved in [14].

Theorem 3.4 Let $\Phi$ any Young function, $\varphi_{1}, \varphi_{2}$, and $\Phi$ satisfy the condition

$$
\sup _{x \in \mathbb{R}^{n}, r>0} \frac{1}{\varphi_{2}(x, r) \Phi^{-1}\left(r^{-n}\right)} \int_{r}^{\infty} \underset{t<s<\infty}{\operatorname{essinf}} \varphi_{1}(x, s) \Phi^{-1}\left(t^{-n}\right) \frac{d t}{t}<\infty,
$$

where $C$ does not depend on $x$ and $r$. Then a $\Phi$-admissible singular operator $T$ is bounded from $M_{\Phi, \varphi_{1}}\left(\mathbb{R}^{n}\right)$ to $M_{\Phi, \varphi_{2}}\left(\mathbb{R}^{n}\right)$ and a weak $\Phi$-admissible singular operator $T$ is bounded from $M_{\Phi, \varphi_{1}}\left(\mathbb{R}^{n}\right)$ to $W M_{\Phi, \varphi_{2}}\left(\mathbb{R}^{n}\right)$.

Theorem 3.5 Let $\Phi$ be a Young function. Let also $\varphi_{1}, \varphi_{1}$, $\Phi$ satisfy the conditions (2.4)-(2.5) and

$$
c_{\delta}:=\int_{\delta}^{\infty} \sup _{x \in \mathbb{R}^{n}} \varphi_{1}(x, t) \frac{\Phi^{-1}\left(t^{-n}\right)}{t} d t<\infty
$$

for every $\delta>0$, and

$$
\frac{1}{\varphi_{2}(x, r) \Phi^{-1}\left(r^{-n}\right)} \int_{r}^{\infty} \varphi_{1}(x, t) \Phi^{-1}\left(t^{-n}\right) \frac{d t}{t} \leq C_{0},
$$

where $C_{0}$ does not depend on $x \in \mathbb{R}^{n}$ and $r>0$. Then a $\Phi$-admissible singular operator $T$ is bounded from $V M_{\Phi, \varphi_{1}}\left(\mathbb{R}^{n}\right)$ to $V M_{\Phi, \varphi_{2}}\left(\mathbb{R}^{n}\right)$ and a weak $\Phi$-admissible singular operator $T$ is bounded from $V M_{\Phi, \varphi_{1}}\left(\mathbb{R}^{n}\right)$ to $V W M_{\Phi, \varphi_{2}}\left(\mathbb{R}^{n}\right)$.

Proof The statement is derived from Theorem 3.4.

So we only have to prove that

$$
\lim _{r \rightarrow 0} \sup _{x \in \mathbb{R}^{n}} \varphi_{1}(x, r)^{-1}\|f\|_{L_{\Phi}(B(x, r))}=0 \Rightarrow \lim _{r \rightarrow 0} \sup _{x \in \mathbb{R}^{n}} \varphi_{2}(x, r)^{-1}\|T f\|_{L_{\Phi}(B(x, r))}=0,
$$

and

$$
\lim _{r \rightarrow 0} \sup _{x \in \mathbb{R}^{n}} \varphi_{1}(x, r)^{-1}\|f\|_{L_{\Phi}(B(x, r))}=0 \quad \Rightarrow \quad \lim _{r \rightarrow 0} \sup _{x \in \mathbb{R}^{n}} \varphi_{2}(x, r)^{-1}\|T f\|_{W L_{\Phi}(B(x, r))}=0 .
$$

In this estimation we follow some ideas of [26] in such a passage to the limit in the case $\Phi(r)=r^{p}$, but we base ourselves on Lemma 3.3.

To show that $\sup _{x \in \mathbb{R}^{n}} \varphi_{2}(x, r)^{-1}\|T f\|_{L_{\Phi}(B(x, r))}<\varepsilon$ for small $r$, we split the right-hand side of (3.1):

$$
\varphi_{2}(x, r)^{-1}\|T f\|_{L_{\Phi}(B(x, r))} \leq C\left[I_{\delta}(x, r)+J_{\delta}(x, r)\right],
$$

where $\delta_{0}>0$ (we may take $\delta_{0}<1$ ), and

$$
I_{\delta}(x, r):=\frac{1}{\Phi^{-1}\left(r^{-n}\right) \varphi_{2}(x, r)}\left(\int_{r}^{\delta_{0}} \frac{\varphi_{1}(x, t) \Phi^{-1}\left(t^{-n}\right)}{t}\left(\varphi_{1}(x, t)^{-1}\|f\|_{L_{\Phi}(B(x, t))}\right) d t\right)
$$


and

$$
J_{\delta}(x, r):=\frac{1}{\Phi^{-1}\left(r^{-n}\right) \varphi_{2}(x, r)}\left(\int_{\delta_{0}}^{\infty} \frac{\varphi_{1}(x, t) \Phi^{-1}\left(t^{-n}\right)}{t}\left(\varphi_{1}(x, t)^{-1}\|f\|_{L_{\Phi}(B(x, t))}\right) d t\right)
$$

and it is supposed that $r<\delta_{0}$. Now we choose any fixed $\delta_{0}>0$ such that

$$
\sup _{x \in \mathbb{R}^{n}} \varphi_{1}(x, t)^{-1}\|f\|_{L_{\Phi}(B(x, t))}<\frac{\varepsilon}{2 C C_{0}}
$$

where $C$ and $C_{0}$ are constants from (3.6) and (3.3). This allows one to estimate the first term uniformly in $r \in\left(0, \delta_{0}\right)$ :

$$
\sup _{x \in \mathbb{R}^{n}} C I_{\delta_{0}}(x, r)<\frac{\varepsilon}{2}, \quad 0<r<\delta_{0} .
$$

The estimation of the second term now may be made already by choosing $r$ sufficiently small. Indeed, thanks to the condition (2.4) we have

$$
J_{\delta}(x, r) \leq c_{\delta_{0}}\|f\|_{V M_{\Phi, \varphi}} \frac{1}{\Phi^{-1}\left(r^{-n}\right) \varphi(x, r)}
$$

where $c_{\delta_{0}}$ is the constant from (3.2). Then by (2.4) it suffices to choose $r$ small enough such that

$$
\sup _{x \in \mathbb{R}^{n}} \frac{1}{\Phi^{-1}\left(r^{-n}\right) \varphi(x, r)} \leq \frac{\varepsilon}{2 c_{\delta_{0}}\|f\|_{V M_{\Phi, \varphi}}}
$$

which completes the proof of (3.4).

The proof of (3.5) is similar to the proof of (3.4).

Remark 3.6 The condition (3.2) is not needed in the case where $\varphi(x, r)$ does not depend on $x$, since (3.2) follows from (3.3) in these cases.

Remark 3.7 Note that from Theorems 3.1 and 3.2 that it is found that the maximal operator $M$ and the singular integral operator $K$ are the weak $\Phi$-admissible singular operators for any Young function $\Phi$ and $\Phi \in \Delta_{2}$, respectively. Also the maximal operator $M$ and the singular integral operator $K$ are the $\Phi$-admissible singular operators for the Young functions $\Phi \in \nabla_{2}$ and $\Phi \in \Delta_{2} \cap \nabla_{2}$, respectively.

From Remark 3.7 we get the following corollaries which were proved in [36].

Corollary 3.8 Let $\Phi$ be a Young function, $\varphi_{1}, \varphi_{2}$, and $\Phi$ satisfy the conditions (2.4)(2.5) and (3.2)-(3.3). Then the maximal operator $M$ is bounded from $V M_{\Phi, \varphi_{1}}\left(\mathbb{R}^{n}\right)$ to $V W M_{\Phi, \varphi_{2}}\left(\mathbb{R}^{n}\right)$ and for $\Phi \in \nabla_{2}$, the operator $M$ is bounded from $V M_{\Phi, \varphi_{1}}\left(\mathbb{R}^{n}\right)$ to $V M_{\Phi, \varphi_{2}}\left(\mathbb{R}^{n}\right)$.

Corollary 3.9 Let $\Phi$ be a Young function, $K$ be a Calderon-Zygmund singular operator with standard kernel and $\varphi_{1}, \varphi_{1}$, $\Phi$ satisfy the conditions (2.4)-(2.5) and (3.2)-(3.3). If $\Phi \in$ $\Delta_{2} \cap \nabla_{2}$, then the operator $K$ is bounded from $V M_{\Phi, \varphi_{1}}\left(\mathbb{R}^{n}\right)$ to $V M_{\Phi, \varphi_{2}}\left(\mathbb{R}^{n}\right)$ and if $\Phi \in \Delta_{2}$, then the operator $K$ is bounded from $V M_{\Phi, \varphi_{1}}\left(\mathbb{R}^{n}\right)$ to $V W M_{\Phi, \varphi_{2}}\left(\mathbb{R}^{n}\right)$. 


\section{Commutators of the $\Phi$-admissible singular operators in the spaces $M_{\Phi, \varphi}$ and $V M_{\Phi, \varphi}$}

It is well known that the commutator is an important integral operator and plays a key role in harmonic analysis. In 1965, Calderon $[37,38]$ studied a kind of commutators appearing in Cauchy integral problems of Lip-line. Let $K$ be a Calderón-Zygmund singular integral operator and $b \in B M O\left(\mathbb{R}^{n}\right)$. A well-known result of Coifman et al. [39] states that the commutator operator $[b, K] f=K(b f)-b K f$ is bounded on $L_{p}\left(\mathbb{R}^{n}\right)$ for $1<p<\infty$. The commutator of Calderón-Zygmund operators plays an important role in studying the regularity of solutions of elliptic partial differential equations of second order. The boundedness result was generalized to other contexts and important applications to some non-linear PDEs were given by Coifman et al. [40].

We recall the definition of the space of $B M O\left(\mathbb{R}^{n}\right)$.

Definition 4.1 Suppose that $b \in L_{1}^{\text {loc }}\left(\mathbb{R}^{n}\right)$, let

$$
\|b\|_{*}=\sup _{x \in \mathbb{R}^{n}, r>0} \frac{1}{|B(x, r)|} \int_{B(x, r)}\left|b(y)-b_{B(x, r)}\right| d y<\infty
$$

where

$$
b_{B(x, r)}=\frac{1}{|B(x, r)|} \int_{B(x, r)} b(y) d y .
$$

Define

$$
B M O\left(\mathbb{R}^{n}\right)=\left\{b \in L_{1}^{\text {loc }}\left(\mathbb{R}^{n}\right):\|b\|_{*}<\infty\right\} .
$$

Modulo constants, the space $B M O\left(\mathbb{R}^{n}\right)$ is a Banach space with respect to the norm $\|\cdot\|_{*}$.

\section{Remark 4.2}

(1) The John-Nirenberg inequality states that there are constants $C_{1}, C_{2}>0$, such that for all $f \in B M O\left(\mathbb{R}^{n}\right)$ and $\beta>0$

$$
\left|\left\{x \in B:\left|b(x)-b_{B}\right|>\beta\right\}\right| \leq C_{1}|B| e^{-C_{2} \beta /\|b\|_{*}}, \quad \forall B \subset \mathbb{R}^{n} .
$$

(2) The John-Nirenberg inequality implies that

$$
\|b\|_{*} \approx \sup _{x \in \mathbb{R}^{n}, r>0}\left(\frac{1}{|B(x, r)|} \int_{B(x, r)}\left|b(y)-b_{B(x, r)}\right|^{p} d y\right)^{\frac{1}{p}}
$$

for $1<p<\infty$.

(3) Let $b \in B M O\left(\mathbb{R}^{n}\right)$. Then there is a constant $C>0$ such that

$$
\left|b_{B(x, r)}-b_{B(x, t)}\right| \leq C\|b\|_{*} \ln \frac{t}{r} \text { for } 0<2 r<t,
$$

where $C$ is independent of $b, x, r$, and $t$.

Definition 4.3 A Young function $\Phi$ is said to be of upper type $p$ (resp. lower type $p$ ) for some $p \in[0, \infty)$, if there exists a positive constant $C$ such that, for all $t \in[1, \infty)$ (resp. 
$t \in[0,1])$ and $s \in[0, \infty)$

$$
\Phi(s t) \leq C t^{p} \Phi(s)
$$

Remark 4.4 We know that if $\Phi$ is lower type $p_{0}$ and upper type $p_{1}$ with $1<p_{0} \leq p_{1}<\infty$, then $\Phi \in \Delta_{2} \cap \nabla_{2}$. Conversely if $\Phi \in \Delta_{2} \cap \nabla_{2}$, then $\Phi$ is lower type $p_{0}$ and upper type $p_{1}$ with $1<p_{0} \leq p_{1}<\infty$ (see [20]).

In the following lemma, which was proved in [13], we provide a generalization of the property (4.1) from $L_{p}$-norms to Orlicz norms.

Lemma 4.5 Let $b \in B M O$ and $\Phi$ be a Young function. Let $\Phi$ is lower type $p_{0}$ and upper type $p_{1}$ with $1 \leq p_{0} \leq p_{1}<\infty$, then

$$
\|b\|_{*} \approx \sup _{x \in \mathbb{R}^{n}, r>0} \Phi^{-1}\left(r^{-n}\right)\left\|b(\cdot)-b_{B(x, r)}\right\|_{L_{\Phi}(B(x, r))} .
$$

Remark 4.6 Note that the Lemma 4.5 for the variable exponent Lebesgue space $L_{p(\cdot)}$ case was proved in [41].

Definition 4.7 Let $\Phi$ be a Young function. Let

$$
a_{\Phi}:=\inf _{t \in(0, \infty)} \frac{t \Phi^{\prime}(t)}{\Phi(t)}, \quad b_{\Phi}:=\sup _{t \in(0, \infty)} \frac{t \Phi^{\prime}(t)}{\Phi(t)} .
$$

Remark 4.8 It is well known that $\Phi \in \Delta_{2} \cap \nabla_{2}$ if and only if $1<a_{\Phi} \leq b_{\Phi}<\infty$ (see, for example, [21]).

Remark 4.9 Remark 4.8 and Remark 4.4 show us that a Young function $\Phi$ is lower type $p_{0}$ and upper type $p_{1}$ with $1<p_{0} \leq p_{1}<\infty$ if and only if $1<a_{\Phi} \leq b_{\Phi}<\infty$.

Definition 4.10 ( $\Phi$-admissible commutator singular operator) Let $\Phi$ any Young function. For a function $b$, the sublinear commutator operator $T_{b}$ will be called a $\Phi$-admissible commutator singular operator, if:

(1) $T_{b}$ satisfies the size condition of the form

$$
\chi_{B(x, r)}(z)\left|T_{b}\left(f \chi_{\mathbb{R}^{n} \backslash B(x, 2 r)}\right)(z)\right| \leq C \chi_{B(x, r)}(z) \int_{\mathbb{R}^{n} \backslash B(x, 2 r)} \frac{|b(y)-b(z)||f(y)|}{|y-z|^{n}} d y
$$

for $x \in \mathbb{R}^{n}$ and $r>0$;

(2) $T_{b}$ is bounded in $L_{\Phi}\left(\mathbb{R}^{n}\right)$.

In the case $\Phi(r)=r^{p}, 1<p<\infty$, the $\Phi$-admissible commutator singular operator will be called a $p$-admissible commutator singular operator.

We will use the following statement on the boundedness of the weighted Hardy operator:

$$
H_{w}^{*} g(r):=\int_{r}^{\infty}\left(1+\ln \frac{t}{r}\right) g(t) w(t) d t, \quad r \in(0, \infty)
$$

where $w$ is a weight. 
The following theorem was proved in [42].

Theorem 4.11 Let $v_{1}, v_{2}$, and $w$ be weights on $(0, \infty)$ and $v_{1}(t)$ be bounded outside a neighborhood of the origin. The inequality

$$
\sup _{r>0} v_{2}(r) H_{w}^{*} g(r) \leq C \sup _{r>0} v_{1}(r) g(r)
$$

holds for some $C>0$ for all non-negative and non-decreasing $g$ on $(0, \infty)$ if and only if

$$
B:=\sup _{r>0} v_{2}(r) \int_{r}^{\infty}\left(1+\ln \frac{t}{r}\right) \frac{w(t) d t}{\sup _{t<s<\infty} v_{1}(s)}<\infty
$$

Moreover, the value $C=B$ is the best constant for (4.3).

Remark 4.12 In (4.3) and (4.4) it is assumed that $\frac{1}{\infty}=0$ and $0 \cdot \infty=0$.

Lemma 4.13 Let $\Phi$ be a Young function with $1<a_{\Phi} \leq b_{\Phi}<\infty, b \in B M O\left(\mathbb{R}^{n}\right), T_{b}$ be $a$ $\Phi$-admissible commutator singular operator, then the inequality

$$
\left\|T_{b} f\right\|_{L_{\Phi}\left(B\left(x_{0}, r\right)\right)} \lesssim \frac{\|b\|_{*}}{\Phi^{-1}\left(r^{-n}\right)} \int_{2 r}^{\infty}\left(1+\ln \frac{t}{r}\right)\|f\|_{L_{\Phi}\left(B\left(x_{0}, t\right)\right)} \Phi^{-1}\left(t^{-n}\right) \frac{d t}{t}
$$

holds for any ball $B\left(x_{0}, r\right)$ and for all $f \in L_{\Phi}^{\text {loc }}\left(\mathbb{R}^{n}\right)$.

Proof For arbitrary $x_{0} \in \mathbb{R}^{n}$, set $B=B\left(x_{0}, r\right)$ for the ball centered at $x_{0}$ and of radius $r$. Write $f=f_{1}+f_{2}$ with $f_{1}=f \chi_{2 B}$ and $f_{2}=f \chi_{\mathrm{c}_{(2 B)}}$. Hence

$$
\left\|T_{b} f\right\|_{L_{\Phi}(B)} \leq\left\|T_{b} f_{1}\right\|_{L_{\Phi}(B)}+\left\|T_{b} f_{2}\right\|_{L_{\Phi}(B)} .
$$

From the boundedness of $T_{b}$ in $L_{\Phi}\left(\mathbb{R}^{n}\right)$ it follows that

$$
\begin{aligned}
\left\|T_{b} f_{1}\right\|_{L_{\Phi}(B)} & \leq\left\|T_{b} f_{1}\right\|_{L_{\Phi}\left(\mathbb{R}^{n}\right)} \\
& \lesssim\|b\|_{*}\left\|f_{1}\right\|_{L_{\Phi}\left(\mathbb{R}^{n}\right)}=\|b\|_{*}\|f\|_{L_{\Phi}(2 B)} .
\end{aligned}
$$

For $x \in B$ we have

$$
\begin{aligned}
\left|T_{b} f_{2}(x)\right| & \lesssim \int_{\mathbb{R}^{n}} \frac{|b(y)-b(x)|}{|x-y|^{n}}|f(y)| d y \\
& \approx \int_{\mathrm{C}_{(2 B)}} \frac{|b(y)-b(x)|}{\left|x_{0}-y\right|^{n}}|f(y)| d y .
\end{aligned}
$$

Then

$$
\begin{aligned}
\left\|T_{b} f_{2}\right\|_{L_{\Phi}(B)} & \lesssim\left\|\int_{\mathrm{C}_{(2 B)}} \frac{|b(y)-b(\cdot)|}{\left|x_{0}-y\right|^{n}}|f(y)| d y\right\|_{L_{\Phi}(B)} \\
& \lesssim\left\|\int_{\mathrm{C}_{(2 B)}} \frac{\left|b(y)-b_{B}\right|}{\left|x_{0}-y\right|^{n}}|f(y)| d y\right\|_{L_{\Phi}(B)}
\end{aligned}
$$




$$
\begin{aligned}
& +\left\|\int_{\mathrm{C}_{(2 B)}} \frac{\left|b(\cdot)-b_{B}\right|}{\left|x_{0}-y\right|^{n}}|f(y)| d y\right\|_{L_{\Phi}(B)} \\
= & I_{1}+I_{2} .
\end{aligned}
$$

Let us estimate $I_{1}$ :

$$
\begin{aligned}
I_{1} & \approx \frac{1}{\Phi^{-1}\left(r^{-n}\right)} \int_{\mathrm{C}_{(2 B)}} \frac{\left|b(y)-b_{B}\right|}{\left|x_{0}-y\right|^{n}}|f(y)| d y \\
& \approx \frac{1}{\Phi^{-1}\left(r^{-n}\right)} \int_{\mathrm{C}_{(2 B)}}\left|b(y)-b_{B}\right||f(y)| \int_{\left|x_{0}-y\right|}^{\infty} \frac{d t}{t^{n+1}} d y \\
& \approx \frac{1}{\Phi^{-1}\left(r^{-n}\right)} \int_{2 r}^{\infty} \int_{2 r \leq\left|x_{0}-y\right| \leq t}\left|b(y)-b_{B}\right||f(y)| d y \frac{d t}{t^{n+1}} \\
& \lesssim \frac{1}{\Phi^{-1}\left(r^{-n}\right)} \int_{2 r}^{\infty} \int_{B\left(x_{0}, t\right)}\left|b(y)-b_{B}\right||f(y)| d y \frac{d t}{t^{n+1}} .
\end{aligned}
$$

Applying Hölder's inequality, by Lemma 4.5 and (4.2) we get

$$
\begin{aligned}
I_{1} \lesssim & \frac{1}{\Phi^{-1}\left(r^{-n}\right)} \int_{2 r}^{\infty} \int_{B\left(x_{0}, t\right)}\left|b(y)-b_{B\left(x_{0}, t\right)}\right||f(y)| d y \frac{d t}{t^{n+1}} \\
& +\frac{1}{\Phi^{-1}\left(r^{-n}\right)} \int_{2 r}^{\infty}\left|b_{B\left(x_{0}, r\right)}-b_{B\left(x_{0}, t\right)}\right| \int_{B\left(x_{0}, t\right)}|f(y)| d y \frac{d t}{t^{n+1}} \\
\lesssim & \frac{1}{\Phi^{-1}\left(r^{-n}\right)} \int_{2 r}^{\infty}\left\|b(\cdot)-b_{B\left(x_{0}, t\right)}\right\|_{L_{\widetilde{\Phi}}\left(B\left(x_{0}, t\right)\right)}\|f\|_{L_{\Phi}\left(B\left(x_{0}, t\right)\right)} \frac{d t}{t^{n+1}} \\
& +\frac{1}{\Phi^{-1}\left(r^{-n}\right)} \int_{2 r}^{\infty}\left|b_{B\left(x_{0}, r\right)}-b_{B\left(x_{0}, t\right)}\right|\|f\|_{L_{\Phi}\left(B\left(x_{0}, t\right)\right)} \Phi^{-1}\left(t^{-n}\right) \frac{d t}{t} \\
\lesssim & \frac{\|b\|_{*}}{\Phi^{-1}\left(r^{-n}\right)} \int_{2 r}^{\infty}\left(1+\ln \frac{t}{r}\right)\|f\|_{L_{\Phi}\left(B\left(x_{0}, t\right)\right)} \Phi^{-1}\left(t^{-n}\right) \frac{d t}{t} .
\end{aligned}
$$

In order to estimate $I_{2}$ note that

$$
I_{2} \approx\left\|b(\cdot)-b_{B}\right\|_{L_{\Phi}(B)} \int_{\mathrm{C}_{(2 B)}} \frac{|f(y)|}{\left|x_{0}-y\right|^{n}} d y .
$$

By Lemma 4.5, we get

$$
I_{2} \lesssim \frac{\|b\|_{*}}{\Phi^{-1}\left(r^{-n}\right)} \int_{\mathrm{C}_{(2 B)}} \frac{|f(y)|}{\left|x_{0}-y\right|^{n}} d y .
$$

On the other hand by Fubini's theorem we have

$$
\begin{aligned}
\int_{\mathrm{C}_{(2 B)}} \frac{|f(y)|}{\left|x_{0}-y\right|^{n}} d y & \approx \int_{\mathrm{C}_{(2 B)}}|f(y)| \int_{\left|x_{0}-y\right|}^{\infty} \frac{d t}{t^{n+1}} d y \\
& \approx \int_{2 r}^{\infty} \int_{2 r \leq\left|x_{0}-y\right|<t}|f(y)| d y \frac{d t}{t^{n+1}} \\
& \lesssim \int_{2 r}^{\infty} \int_{B\left(x_{0}, t\right)}|f(y)| d y \frac{d t}{t^{n+1}}
\end{aligned}
$$


By Lemma 2.6 we get

$$
\int_{\mathrm{C}_{(2 B)}} \frac{|f(y)|}{\left|x_{0}-y\right|^{n}} d y \lesssim \int_{2 r}^{\infty}\|f\|_{L_{\Phi}\left(B\left(x_{0}, t\right)\right)} \Phi^{-1}\left(t^{-n}\right) \frac{d t}{t} .
$$

Therefore using (4.7) at (4.6) we have

$$
I_{2} \lesssim \frac{\|b\|_{*}}{\Phi^{-1}\left(r^{-n}\right)} \int_{2 r}^{\infty}\|f\|_{L_{\Phi}\left(B\left(x_{0}, t\right)\right)} \Phi^{-1}\left(t^{-n}\right) \frac{d t}{t}
$$

Summing $I_{1}$ and $I_{2}$, we obtain

$$
\left\|T_{b} f_{2}\right\|_{L_{\Phi}(B)} \lesssim \frac{\|b\|_{*}}{\Phi^{-1}\left(r^{-n}\right)} \int_{2 r}^{\infty}\left(1+\ln \frac{t}{r}\right)\|f\|_{L_{\Phi}\left(B\left(x_{0}, t\right)\right)} \Phi^{-1}\left(t^{-n}\right) \frac{d t}{t} .
$$

On the other hand, by (2.2) we get

$$
\Phi^{-1}\left(r^{-n}\right) \approx \Phi^{-1}\left(r^{-n}\right) r^{n} \int_{2 r}^{\infty} \frac{d t}{t^{n+1}} \lesssim \int_{2 r}^{\infty} \Phi^{-1}\left(t^{-n}\right) \frac{d t}{t}
$$

and then

$$
\|f\|_{L_{\Phi}(2 B)} \lesssim \frac{1}{\Phi^{-1}\left(r^{-n}\right)} \int_{2 r}^{\infty}\|f\|_{L_{\Phi}\left(B\left(x_{0}, t\right)\right)} \Phi^{-1}\left(t^{-n}\right) \frac{d t}{t}
$$

Finally,

$$
\begin{aligned}
\left\|T_{b} f\right\|_{L_{\Phi}(B)} \lesssim & \|b\|_{*}\|f\|_{L_{\Phi}(2 B)} \\
& +\frac{\|b\|_{*}}{\Phi^{-1}\left(r^{-n}\right)} \int_{2 r}^{\infty}\left(1+\ln \frac{t}{r}\right)\|f\|_{L_{\Phi}\left(B\left(x_{0}, t\right)\right)} \Phi^{-1}\left(t^{-n}\right) \frac{d t}{t},
\end{aligned}
$$

and the statement of Lemma 4.13 follows by (4.9).

Theorem 4.14 Let $\Phi$ be a Young function with $1<a_{\Phi} \leq b_{\Phi}<\infty, b \in B M O\left(\mathbb{R}^{n}\right), T_{b}$ be $a$ $\Phi$-admissible commutator singular operator, $\varphi_{1}, \varphi_{2}$, and $\Phi$ satisfy the condition

$$
\int_{r}^{\infty}\left(1+\ln \frac{t}{r}\right)\left(\underset{t<s<\infty}{\operatorname{essinf}} \varphi_{1}(x, s)\right) \Phi^{-1}\left(t^{-n}\right) \frac{d t}{t} \leq C \varphi_{2}(x, r) \Phi^{-1}\left(r^{-n}\right),
$$

where $C$ does not depend on $x$ and $r$. Then the operator $T_{b}$ is bounded from $M_{\Phi, \varphi_{1}}\left(\mathbb{R}^{n}\right)$ to $M_{\Phi, \varphi_{2}}\left(\mathbb{R}^{n}\right)$. Moreover,

$$
\left\|T_{b} f\right\|_{M_{\Phi, \varphi_{2}}} \lesssim\|b\|_{*}\|f\|_{M_{\Phi, \varphi_{1}}} .
$$

Proof The statement of Theorem 4.14 follows by Lemma 4.13 and Theorem 4.11 in the same manner as in the proof of Theorem 3.4.

If we take $\Phi(r)=r^{p}, 1<p<\infty$ at Theorem 4.14 we get the following result, which was proved at [43]. 
Corollary 4.15 Let $1<p<\infty, b \in B M O\left(\mathbb{R}^{n}\right), T_{b}$ be a $p$-admissible commutator singular operator and $\left(\varphi_{1}, \varphi_{2}\right)$ satisfies the condition

$$
\int_{r}^{\infty}\left(1+\ln \frac{t}{r}\right) \frac{\operatorname{essinf}_{t<s<\infty} \varphi_{1}(x, s)}{t^{\frac{n}{p}+1}} d t \leq C \frac{\varphi_{2}(x, r)}{r^{\frac{n}{p}}}
$$

where $C$ does not depend on $x$ and $r$. Then the operator $T_{b}$ is bounded from $M_{p, \varphi_{1}}\left(\mathbb{R}^{n}\right)$ to $M_{p, \varphi_{2}}\left(\mathbb{R}^{n}\right)$.

The commutator of the maximal operator is defined by

$$
M_{b}(f)(x)=\sup _{t>0}|B(x, t)|^{-1} \int_{B(x, t)}|b(x)-b(y)||f(y)| d y .
$$

The known boundedness statement for the commutator operators $[b, K]$ and $M_{b}$ on Orlicz spaces runs as follows.

Theorem 4.16 [44] Let $\Phi$ be a Young function with $1<a_{\Phi} \leq b_{\Phi}<\infty, b \in B M O\left(\mathbb{R}^{n}\right)$. Then the operators $[b, K]$ and $M_{b}$ are bounded on $L_{\Phi}\left(\mathbb{R}^{n}\right)$.

For the commutator operators $[b, K]$ and $M_{b}$ from Theorem 4.14 we get the following corollaries, which were proved in [36].

Corollary 4.17 Let $\Phi$ be a Young function with $1<a_{\Phi} \leq b_{\Phi}<\infty, b \in B M O\left(\mathbb{R}^{n}\right)$ and $\varphi_{1}$, $\varphi_{2}$, and $\Phi$ satisfy the condition (4.10). Then the operators $[b, K]$ and $M_{b}$ are bounded from $M_{\Phi, \varphi_{1}}\left(\mathbb{R}^{n}\right)$ to $M_{\Phi, \varphi_{2}}\left(\mathbb{R}^{n}\right)$.

Theorem 4.18 Let $\Phi$ be a Young function with $1<a_{\Phi} \leq b_{\Phi}<\infty, b \in B M O\left(\mathbb{R}^{n}\right), T_{b}$ be a $\Phi$-admissible commutator singular operator. Let also $\varphi_{1}, \varphi_{2}$, $\Phi$ satisfy

$$
\int_{r}^{\infty}\left(1+\ln \frac{t}{r}\right) \varphi_{1}(x, t) \Phi^{-1}\left(t^{-n}\right) \frac{d t}{t} \leq C_{0} \varphi_{2}(x, r) \Phi^{-1}\left(r^{-n}\right)
$$

where $C_{0}$ does not depend on $x \in \mathbb{R}^{n}$ and $r>0$, and the conditions

$$
\lim _{r \rightarrow 0} \frac{\ln \frac{1}{r}}{\Phi^{-1}\left(r^{-n}\right) \inf _{x \in \mathbb{R}^{n}} \varphi_{2}(x, r)}=0
$$

and

$$
c_{\delta}:=\int_{\delta}^{\infty}(1+|\ln t|) \sup _{x \in \mathbb{R}^{n}} \varphi_{1}(x, t) \frac{\Phi^{-1}\left(t^{-n}\right)}{t} d t<\infty
$$

for every $\delta>0$. Then the operator $T_{b}$ is bounded from $V M_{\Phi, \varphi_{1}}\left(\mathbb{R}^{n}\right)$ to $V M_{\Phi, \varphi_{2}}\left(\mathbb{R}^{n}\right)$.

Proof The proof follows more or less the same lines as for Theorem 3.5, but now the arguments are different due to the necessity to introduce the logarithmic factor into the assumptions. 
The norm inequality having already been provided by Theorem 4.14, we only have to prove the implication

$$
\lim _{r \rightarrow 0} \sup _{x \in \mathbb{R}^{n}} \frac{\|f\|_{L_{\Phi}(B(x, r))}}{\varphi_{1}(x, r)}=0 \Longrightarrow \lim _{r \rightarrow 0} \sup _{x \in \mathbb{R}^{n}} \frac{\left\|T_{b} f\right\|_{L_{\Phi}(B(x, r))}}{\varphi_{2}(x, r)}=0 .
$$

To check that

$$
\sup _{x \in \mathbb{R}^{n}} \frac{\left\|T_{b} f\right\|_{L_{\Phi}(B(x, r))}}{\varphi_{2}(x, r)}<\varepsilon \quad \text { for small } r,
$$

we use the estimate (4.5):

$$
\frac{\left\|T_{b} f\right\|_{L_{\Phi}(B(x, r))}}{\varphi_{2}(x, r)} \lesssim \frac{\|b\|_{*}}{\varphi_{2}(x, r) \Phi^{-1}\left(r^{-n}\right)} \int_{r}^{\infty}\left(1+\ln \frac{t}{r}\right)\|f\|_{L_{\Phi}\left(B\left(x_{0}, t\right)\right)} \Phi^{-1}\left(t^{-n}\right) \frac{d t}{t} .
$$

We take $r<\delta_{0}$ where $\delta_{0}$ will be chosen small enough and we split the integration:

$$
\frac{\left\|T_{b} f\right\|_{L_{\Phi}(B(x, r))}}{\varphi_{2}(x, r)} \leq C\left[I_{\delta_{0}}(x, r)+J_{\delta_{0}}(x, r)\right]
$$

where

$$
I_{\delta_{0}}(x, r):=\frac{1}{\Phi^{-1}\left(r^{-n}\right) \varphi_{2}(x, r)} \int_{r}^{\delta_{0}}\left(1+\ln \frac{t}{r}\right) \frac{\Phi^{-1}\left(t^{-n}\right)}{t}\|f\|_{L_{\Phi}(B(x, t))} d t
$$

and

$$
J_{\delta_{0}}(x, r):=\frac{1}{\Phi^{-1}\left(r^{-n}\right) \varphi_{2}(x, r)} \int_{\delta_{0}}^{\infty}\left(1+\ln \frac{t}{r}\right) \frac{\Phi^{-1}\left(t^{-n}\right)}{t}\|f\|_{L_{\Phi}(B(x, t))} d t .
$$

We choose a fixed $\delta_{0}>0$ such that

$$
\sup _{x \in \mathbb{R}^{n}} \frac{\|f\|_{L_{\Phi}(B(x, t))}}{\varphi_{1}(x, t)}<\frac{\varepsilon}{2 C C_{0}}, \quad t \leq \delta_{0},
$$

where $C$ and $C_{0}$ are constants from (4.15) and (4.11), which yields the estimate of the first term uniform in $r \in\left(0, \delta_{0}\right): \sup _{x \in \mathbb{R}^{n}} C I_{\delta_{0}}(x, r)<\frac{\varepsilon}{2}, 0<r<\delta_{0}$.

For the second term, writing $1+\ln \frac{t}{r} \leq 1+|\ln t|+\ln \frac{1}{r}$, we obtain

$$
J_{\delta_{0}}(x, r) \leq \frac{c_{\delta_{0}}+\widetilde{c}_{\delta_{0}} \ln \frac{1}{r}}{\Phi^{-1}\left(r^{-n}\right) \varphi_{2}(x, r)}\|f\|_{M_{\Phi, \varphi}}
$$

where $c_{\delta_{0}}$ is the constant from (4.13) with $\delta=\delta_{0}$ and $\widetilde{c}_{\delta_{0}}$ is a similar constant with omitted logarithmic factor in the integrand. Then by (4.12) we can choose a small $r$ such that $\sup _{x \in \mathbb{R}^{n}} J_{\delta_{0}}(x, r)<\frac{\varepsilon}{2}$, which completes the proof.

Corollary 4.19 [36] Let $\Phi$ be a Young function with $1<a_{\Phi} \leq b_{\Phi}<\infty, b \in B M O\left(\mathbb{R}^{n}\right)$, and $\varphi_{1}, \varphi_{2}$, and $\Phi$ satisfy the conditions (4.11), (4.12), and (4.13). Then the operators $[b, K]$ and $M_{b}$ are bounded from $V M_{\Phi, \varphi_{1}}\left(\mathbb{R}^{n}\right)$ to $V M_{\Phi, \varphi_{2}}\left(\mathbb{R}^{n}\right)$. 


\section{Competing interests}

The authors declare that they have no competing interests.

\section{Authors' contributions}

This work was carried out in collaboration between all authors. VSG raised these interesting problems in the research VSG, FD and JJH proved the theorems, interpreted the results and wrote the article. All authors defined the research theme, and read and approved the manuscript.

\section{Acknowledgements}

The research of $V$ Guliyev and $F$ Deringoz were partially supported by the grant of Ahi Evran University Scientific Research Projects (PYO.FEN.4003.13.003), (PYO.FEN.4003-2.13.007) and (PYO.FEN.4009.14.001). We thank both referees for some good suggestions, which helped to improve the final version of this paper.

Received: 5 November 2013 Accepted: 17 March 2014 Published: 08 Apr 2014

\section{References}

1. Morrey, CB: On the solutions of quasi-linear elliptic partial differential equations. Trans. Am. Math. Soc. 43, 126-166 (1938)

2. Orlicz, W: Über eine gewisse Klasse von Räumen vom Typus B. Bull. Acad. Polon. A, 207-220 (1932). Reprinted in: Collected Papers, PWN, Warszawa, 217-230 (1988)

3. Orlicz, W: Über Räume ( $\left.L^{M}\right)$. Bull. Acad. Polon. A, 93-107 (1936). Reprinted in: Collected Papers, PWN, Warszawa, 345-359 (1988)

4. Kita, H: On maximal functions in Orlicz spaces. Proc. Am. Math. Soc. 124, 3019-3025 (1996)

5. Kita, H: On Hardy-Littlewood maximal functions in Orlicz spaces. Math. Nachr. 183, 135-155 (1997)

6. Cianchi, A: Strong and weak type inequalities for some classical operators in Orlicz spaces. J. Lond. Math. Soc. (2) 60(1), 187-202 (1999)

7. Stein, EM: Harmonic Analysis: Real Variable Methods, Orthogonality and Oscillatory Integrals. Princeton University Press, Princeton (1993)

8. Torchinsky, A: Real Variable Methods in Harmonic Analysis. Pure and Applied Math., vol. 123. Academic Press, New York (1986)

9. Nakai, E: Generalized fractional integrals on Orlicz-Morrey spaces. In: Banach and Function Spaces (Kitakyushu, 2003), pp. 323-333. Yokohama Publishers, Yokohama (2004)

10. Nakai, E: Calderón-Zygmund operators on Orlicz-Morrey spaces and modular inequalities. In: Banach and Function Spaces II, pp. 393-410. Yokohama Publishers, Yokohama (2008)

11. Sawano, Y, Sugano, S, Tanaka, H: Orlicz-Morrey spaces and fractional operators. Potential Anal. 36(4), $517-556$ (2012)

12. Deringoz, F, Guliyev, VS, Samko, S: Boundedness of maximal and singular operators on generalized Orlicz-Morrey spaces. In: Operator Theory, Operator Algebras and Applications. Operator Theory: Advances and Applications, vol. 235, pp. 139-158 (2014)

13. Guliyev, VS, Deringoz, F: On the Riesz potential and its commutators on generalized Orlicz-Morrey spaces. J. Funct. Spaces Appl. 2014, Article ID 617414 (2014)

14. Hasanov, JJ: $\Phi$-admissible sublinear singular operators and generalized Orlicz-Morrey spaces. J. Funct. Spaces Appl. 2014, Article ID 505237 (2014)

15. Liang, Y, Nakai, E, Yang, D, Zhang, J: Boundedness of intrinsic Littlewood-Paley functions on Musielak-Orlicz Morrey and Campanato spaces. Banach J. Math. Anal. 8(1), 221-268 (2014)

16. Lu, Q, Tao, X: Characterization of maximal operators in Orlicz-Morrey spaces of homogeneous type. Appl. Math. J. Chin. Univ. Ser. B 21(1), 52-58 (2006)

17. Maeda, FY, Mizuta, Y, Ohno, T, Shimomura, T: Boundedness of maximal operators and Sobolev's inequality on Musielak-Orlicz-Morrey spaces. Bull. Sci. Math. 137(1), 76-96 (2013)

18. Mizuta, Y, Nakai, E, Ohno, T, Shimomura, T: Maximal functions, Riesz potentials and Sobolev embeddings on Musielak-Orlicz-Morrey spaces of variable exponent in $\mathbb{R}^{n}$. Rev. Mat. Complut. 25(2), 413-434 (2012)

19. Nakai, E: Orlicz-Morrey spaces and the Hardy-Littlewood maximal function. Stud. Math. 188(3), 193-221 (2008)

20. Kokilashvili, V, Krbec, MM: Weighted Inequalities in Lorentz and Orlicz Spaces. World Scientific, Singapore (1991)

21. Krasnoselskii, MA, Rutickii, YB: Convex Functions and Orlicz Spaces. Noordhoff, Groningen (1961). (English translation)

22. Rao, MM, Ren, ZD: Theory of Orlicz Spaces. Dekker, New York (1991)

23. Weiss, G: A note on Orlicz spaces. Port. Math. 15, 35-47 (1956)

24. Bennett, C, Sharpley, R: Interpolation of Operators. Academic Press, Boston (1988)

25. Liu, PD, Wang, MF: Weak Orlicz spaces: some basic properties and their applications to harmonic analysis. Sci. China Math. 56(4), 789-802 (2013)

26. Samko, N: Maximal, potential and singular operators in vanishing generalized Morrey spaces. J. Global Optim. (2014). doi:10.1007/s10898-012-9997-x

27. Vitanza, C: Functions with vanishing Morrey norm and elliptic partial differential equations. In: Proceedings of Methods of Real Analysis and Partial Differential Equations, Capri, pp. 147-150. Springer, Berlin (1990)

28. Vitanza, C: Regularity results for a class of elliptic equations with coefficients in Morrey spaces. Ric. Mat. 42(2), 265-281 (1993)

29. Miranda, C: Sulle equazioni ellittiche del secondo ordine di tipo non variazionale, a coefficienti discontinui. Ann. Mat. Pura Appl. (4) 63, 353-386 (1963)

30. Ragusa, MA: Commutators of fractional integral operators on vanishing-Morrey spaces. J. Glob. Optim. 40(1-3), 361-368 (2008)

31. Persson, LE, Ragusa, MA, Samko, N, Wall, P: Commutators of Hardy operators in vanishing Morrey spaces. In: AIP Conf. Proc., vol. 1493, p. 859 (2012). doi:10.1063/1.4765588

32. Genebashvili, I, Gogatishvili, A, Kokilashvili, V, Krbec, M: Weight Theory for Integral Transforms on Spaces of Homogeneous Type. Longman, Harlow (1998) 
33. Guliyev, VS: Integral operators on function spaces on the homogeneous groups and on domains in $\mathbb{R}^{n}$. Doctor's degree dissertation. Mat. Inst. Steklov, Moscow, 329 pp. (1994) (in Russian)

34. Guliyev, VS: Function spaces, Integral Operators and Two Weighted Inequalities on Homogeneous Groups. Some Applications. Casioglu, Baku, 332 pp. (1999) (in Russian)

35. Guliyev, VS: Boundedness of the maximal, potential and singular operators in the generalized Morrey spaces. J. Inequal. Appl. 2009, Article ID 503948 (2009)

36. Deringoz, F, Guliyev, VS, Samko, S: Boundedness of maximal and singular operators and their commutators on vanishing generalized Orlicz-Morrey spaces (submitted)

37. Calderon, AP: Commutators of singular integral operators. Proc. Natl. Acad. Sci. USA 53, 1092-1099 (1965)

38. Calderon, AP: Cauchy integrals on Lipschitz curves and related operators. Proc. Natl. Acad. Sci. USA 74(4), 1324-1327 (1977)

39. Coifman, RR, Rochberg, R, Weiss, G: Factorization theorems for Hardy spaces in several variables. Ann. Math. (2) 103(3), 611-635 (1976)

40. Coifman, R, Lions, P, Meyer, Y, Semmes, S: Compensated compactness and Hardy spaces. J. Math. Pures Appl. 72 , 247-286 (1993)

41. Izuki, M, Sawano, Y: Variable Lebesgue norm estimates for BMO functions. Czechoslov. Math. J. 62(137)(3), 717-727 (2012)

42. Guliyev, VS: Generalized weighted Morrey spaces and higher order commutators of sublinear operators. Eurasian Math. J. 3(3), 33-61 (2012)

43. Guliyev, VS, Aliyev, SS, Karaman, T, Shukurov, PS: Boundedness of sublinear operators and commutators on generalized Morrey space. Integral Equ. Oper. Theory 71(3), 327-355 (2011)

44. Fu, X, Yang, D, Yuan, W: Boundedness on Orlicz spaces for multilinear commutators of Calderón-Zygmund operators on non-homogeneous spaces. Taiwan. J. Math. 16, 2203-2238 (2012)

10.1186/1029-242X-2014-143

Cite this article as: Guliyev et al.: $\Phi$-Admissible singular operators and their commutators on vanishing generalized Orlicz-Morrey spaces. Journal of Inequalities and Applications 2014, 2014:143

\section{Submit your manuscript to a SpringerOpen ${ }^{\ominus}$ journal and benefit from:}

- Convenient online submission

- Rigorous peer review

Immediate publication on acceptance

- Open access: articles freely available online

- High visibility within the field

- Retaining the copyright to your article 